\title{
Inequality as an Explanation for Obesity in the United States
}

Hedwig Lee*

Department of Epidemiology, School of Public Health, University of Michigan

\begin{abstract}
Over the past several decades, there has been a sharp increase in obesity across all population groups in the United States. In fact, the United States has one of the highest rates of obesity compared to other countries throughout the world. Obesity has become a national public health concern because it is related to a number of negative health, social, psychological, and economic outcomes. It is particularly concerning because racial/ethnic minorities and populations with the least education and highest poverty rates bear the largest burden of obesity. In addition, disparities in obesity tend to be gendered, with women experiencing the largest disparities in obesity by income, education, and race/ethnicity. In this review, I describe how social inequality is linked to obesity in the United States. I highlight elements of disadvantage at the individual-, family-, school-, and neighborhood-level that are linked to energy intake and expenditure, which are directly related to obesity, and draw from evidence and theories from multiple fields of the social and medical sciences. I also highlight the important role stress may play in linking disadvantage to obesity, particularly for women. I argue that understanding the complex mechanisms and processes that link social inequality to obesity requires multidisciplinary and multilevel frameworks.
\end{abstract}

\section{Introduction}

Over the past several decades, there has been a sharp increase in obesity across all population groups in the United States (US). Obesity has increased for both males and females, across all ages and all races/ethnicities (Flegal et al. 2010; NCHS 2008; Ogden and Carroll 2010). In a recent report by the Organisation for Economic Co-Operation and Development (OECD), the United States was determined to be the fattest country, having the highest rates of obesity compared to 39 other OECD and non-OECD countries (2010). According to current US estimates, 33.8 percent of adults (ages 20 and older) are obese (defined as body mass index (BMI) of weight $(\mathrm{kg}) /$ height $\left(\mathrm{m}^{2}\right) \geq 30$; Flegal et al. 2010) and 16.9 percent of children and adolescents (ages 2-19) are obese (BMI $\geq 95$ th percentile of the Center for Disease Control (CDC) growth charts; Ogden et al. 2010a).

Obesity has become a national public health concern because it is related to a number of negative health, social, psychological, and economic outcomes. Obesity is associated with multiple morbidities including type 2 diabetes, metabolic syndrome, hypertension, cardiovascular disease (CVD), high cholesterol, sleep apnea, asthma, osteoarthritis, certain types of cancer, and even death (CDC 2009; Daniels 2006; Dor et al. 2010; Mokdad et al. 2004). Obese individuals are more likely to suffer from depression and low self-esteem, have less friends, and experience discrimination and social stigmatization (Daniels 2006; Dor et al. 2010; Puhl and Heuer 2009). Obesity is also associated with less education, lower wages, and a lower likelihood of marriage, particularly for women (Averett and Korenman 1996; Cawley 2004; Crosnoe 2007; Dor et al. 2010; Gortmaker et al. 1993; 
Swallen et al. 2005; Viner and Cole 2005). In addition, as discussed in the Surgeon General's report on obesity, obesity is associated with increased costs to society via direct health care costs and indirect costs through the value of lost future earnings due to premature death and lost wages due to disability or illness, resulting in restricted activity, decreased productivity, absenteeism, bed days, and unemployment (USHHS 2001). According to a recent review, in the United States, the total annual (direct and indirect) costs attributable to overweight and obesity could be as high as $\$ 139$ billion (Finkelstein et al. 2005).

Similar to other developed countries across the world, in the United States, the largest burden of obesity is born by racial/ethnic minorities and populations with the least education and highest poverty rates (Cummins and McIntyre 2006; Drewnowski and Specter 2004; OECD 2010). Data from the 2007-2008 National Health and Nutrition Examination Surveys (NHANES) shows that among adults (ages 20 and older), 44.1 percent of non-Hispanic Blacks are obese compared to 40.4 percent of Mexican Americans and 32.4 percent of non-Hispanic Whites (Flegal et al. 2010). Among children and adolescents (ages 2-19), 20.0 percent of non-Hispanic Blacks are obese compared to 20.8 percent of Mexican Americans and 15.3 percent of non-Hispanic Whites (Ogden et al. 2010a). Less information is available regarding American Indians, Alaska Natives, Pacific Islander Americans, and Asian Americans. Available information indicates a lower prevalence of overweight and obesity in Asian Americans and a higher prevalence in American Indians, Alaska Natives, and Pacific Islander Americans compared to the general population (Wang and Beydoun 2007). It is important to note that there are complexities in the relationship between race/ethnicity, gender, and obesity. These relationships vary by age and there are important gender differences within racial/ethnic groups that should be considered (Robinson et al. 2009; Wang and Beydoun 2007). For example, the prevalence obesity is higher among females (35.5\%) versus males (32.2\%) in adulthood (ages 20 and older); but the prevalence of obesity is lower among females (15.9\%) versus males $(17.8 \%)$ in childhood/adolescence (ages 2-19) (Flegal et al. 2010; Ogden et al. 2010a). In addition, racial/ethnic differences in obesity prevalence vary when examining patterns within gender, evidencing more pronounced racial/ethnic differences among women. The prevalence of obesity is 49.6 percent among non-Hispanic Black women and 45.1 percent among Mexican American women compared to 33.0 percent among non-Hispanic White women. For men, racial differences are less severe with prevalence at 37.3, 35.9, and 31.9 percent for non-Hispanic Blacks, Mexican Americans, and non-Hispanic Whites, respectively (Flegal et al. 2010). Similar to racial/ethnic differences, obesity is higher among the poor and low educated. However, these relationships are also complicated by race/ethnicity, gender, and age (Clarke et al. 2009a; Miech et al. 2006; Sánchez-Vaznaugh et al. 2009; Wang and Beydoun 2007). For example, although previous research has found negative income and education gradients in BMI among women, these relationships have been less consistent, and sometimes even non-existent, in men. (Ball and Crawford 2005; Molarius et al. 2000; OECD 2010; Sánchez-Vaznaugh et al. 2009; Sobal and Stunkard 1989; Wang and Beydoun 2007). As discussed earlier, females also bear larger economic costs for being obese, in the form of lower wages (Dor et al. 2010). Unlike adults, in general, there are no considerable (or consistent) gender differences in socioeconomic gradients in childhood obesity (OECD 2010; Shrewsbury and Wardle 2008); although patterns do vary by race/ethnicity (Kumanyika and Grier 2006; Ogden et al. 2010b). Despite complexities in the relationship between socioeconomic status (SES) and obesity, these patterns highlight the high risk of obesity for disadvantaged and vulnerable population groups. Social scientists have been interested in describing these relationships, as well as theorizing and 
modeling the mechanisms that might link disadvantage to obesity in both empirical and qualitative work.

This article is organized as follows. I will first describe the different ways that inequality can be measured as it relates to health, highlighting both absolute and relative explanations of inequality. Drawing from largely materialist explanations of inequality, I will then describe how social inequality is linked to obesity in the United States. I will use a social-ecological perspective (Bronfenbrenner 1979, 2005; Dahlgren and Whitehead 1991; Glass and McAtee 2006) to describe how disadvantage at multiple levels of social context might influence obesity outcomes among children, adolescents, and adults through behaviors associated with energy intake (calories taken in from food and beverages) and energy expenditure (calories expended by physical activity and metabolic functions), as well as how these relationships are complicated by stress and gender. Understanding the complex relationship between social inequality and obesity requires drawing from evidence and theories from multiple fields of social science including, but not limited to, sociology, demography, psychology, epidemiology, and other fields of public health and medicine (Huang et al. 2009). The papers I cite and theories that I discuss in this review draw from work in these multiple fields, in addition to sociology, that help to inform this important issue. It should be noted that the rapid rise in obesity that has occurred in the United States in the last 40 years is the result of multiple and cooccurring social, biological, technological, and economic processes (Anderson and Butcher 2006a; Apovian 2010; Philipson and Posner 2008). It is beyond the scope of this review to detail the many complex causes of the rise in obesity for the entire population of the United States, as well as in other countries. Instead this review focuses on the explaining how inequality, measured at multiple levels of social disadvantage, can lead to higher levels of obesity in the United States.

\section{Measuring inequality}

There are multiple ways to conceptualize social inequality as it relates to health outcomes. We can examine absolute or relative differences in income, occupation, wealth, and education (or other markers of SES) (Bartley 2004; Goodman 2003).

In materialist explanations, it is argued that health is influenced by material conditions in life that are the result of absolute differences in individual wealth, income, occupation and education, and other resources. Availability of resources, working and living conditions, as well as access to proper nutrition and medical services, both shape health and influence health behaviors (Bartley 2004; Townsend et al. 1992). For example, poor families may not be able to afford nutritious foods and therefore, must consume cheap unhealthy food that can lead to weight gain. Neo-materialist theories expand upon these materialist explanations by describing the origins of poverty and income inequality (in the form of higher proportions of low-income populations and large gaps in income between the rich and the poor) that lead to poor health (Lynch et al. 2000). In other words, these theories examine the social policies and processes that lead to underinvestment in social, public, and physical infrastructure (e.g., poor housing quality and schools; lack of health insurance, social services and job opportunities) resulting in negative health outcomes for vulnerable populations (Lynch and Kaplan 1997). These historical, cultural, political, and economic processes determine the distribution of economic and social resources in a geographic region to create material conditions that influence health (Lynch et al. 2000). For example, due to local housing policies and real estate practices, as well as other processes, poor and racial/ethnic minority individuals tend to live in 
economically and racially segregated neighborhoods with little to no access to grocery stores providing affordable nutritious foods (Massey and Denton 1993; Morland et al. 2002; Williams and Collins 2001). These conditions further compound the food consumption choices of disadvantaged individuals with limited budgets. It is the combination of multiple negative exposures faced by disadvantaged individuals that generate poor health (Lynch et al. 2000).

Psychosocial interpretations argue that health and mortality are influenced by the relative distribution of wealth and income and not absolute levels. Income inequality is a measure of the magnitude of the gap in income between the poor and the rich. According to a psychosocial interpretation, wider gaps in income inequality can lead to negative psychological changes at the individual level and within a society that result in poor health outcomes (Kawachi and Kennedy 1997a, 1999; Wilkinson 1992, 1996; Wilkinson and Pickett 2006, 2007, 2009a,b). Among individuals, income inequality influences perceptions of social rank and relative position according to income (as well as other markers of social status), which produce negative emotions, such as feelings of social anxiety, shame, envy, frustration, worthlessness, and distrust that are internalized and can result in maladaptive stress-induced coping behaviors, such as smoking or overeating, as well as psycho-neuro-endocrine mechanisms that can lead directly to poor health (Lynch 2000; Lynch et al. 2000; Wilkinson 1996). Within a society, negative emotions fostered by relative positions can also be externalized and lead to reduced civic participation, disinvestment in social capital, loss of social cohesion, and other antisocial behaviors that can also lead to worse health for the entire community (Kawachi and Kennedy 1997a, 1999; Lynch et al. 2000; Wilkinson 1992; Wilkinson and Pickett 2009a,b). The psychosocial interpretation also posits that relative inequality, even among high SES individuals can lead to poor health outcomes (Marmot 2004). For example, consider a situation where two female (associate) lawyers, both single with no children, have worked in a law firm for the same number of years and made the same amount of money $(\$ 180,000$ per year) but one makes partner and her income increases to $\$ 250,000$, while the other lawyer neither becomes partner nor receives a raise. A psychosocial interpretation would conjecture that the lawyer who did not make partner would have poorer health than the lawyer who did make partner because her perceptions of lower social status compared to the other lawyer who made partner would engender negative emotions that would lead to disease-causing processes discussed above. Although both lawyers make upper middle class incomes and have similar life circumstances, the non-partnered lawyer would experience poor health and possibly a shorter life expectancy because her status is lower and possessions fewer than the partnered lawyer.

In summary, neomaterialist and materialist explanations for the link between inequality and health focus on the structural causes of inequalities and material conditions that lead to poor health, whereas psychosocial explanations for the link between inequality and health focus on perceptions of inequality that lead to poor health (Lynch et al. 2000). It should be noted that although there are multiple ways to measure inequality and the relationship between inequality and health is complex (i.e., occurring via multiple and unique pathways) (Beckfield and Krieger 2009), in general, greater inequality is associated with greater mortality and poorer health, no matter how it is measured (Goodman 2003; Kawachi and Kennedy 1997b; Kondo et al. 2009; Robert 1999; Wilkinson and Pickett 2009a,b). For a noteworthy exception, read Beckfield (2004).

In this article, I will focus on research that examines absolute differences in income, particularly poverty, wealth, and education at multiple levels of social context and how they relate to obesity. I will draw from a large body of multidisciplinary research that examines the relationship between social disadvantage and obesity outcomes, using mea- 
sures of disadvantage at the individual-, family-, school-, and neighborhood-level (e.g., Boardman et al. 2005; Clarke et al. 2009a; Janssen et al. 2006; Lee et al. 2009; Lovasi et al. 2009; McLaren 2007; Miech et al. 2006; Mujahid et al. 2005; Richmond and Subramanian 2008; Robinson et al. 2009; Scharoun-Lee et al. 2009; Senese et al. 2009; Wickrama et al. 2006; Ziol-Guest et al. 2009). There is also research that examines the relationship between relative measures of income inequality and obesity (see, e.g., Chang and Christakis 2005; Harper and Lynch 2007; Lemeshow et al. 2008; Kima et al. 2008; Pickett et al. 2005; Wilkinson and Pickett 2009b; Zhang and Wang 2004). Although this is also an important area of research, I review research that has examined the specific role material conditions play in influencing obesity status. Future reviews should be devoted to a discussion of research examining the relationship between relative measures of inequality and obesity which also deserves attention.

\section{Inequality and health}

At every point along the life course, social disadvantage (or lower SES) is associated with higher mortality and worse health (Adler and Ostrove 1999; Elo 2009; Feinstein 1993; Lutfey and Freese 2005; Robert and House 1994). Social disadvantage, measured in terms of low education, low occupational status, poverty, and race/ethnicity are social conditions that represent access to resources, such as medical care and information regarding health risks and health care, as well as exposure to certain environmental conditions that are not beneficial to good health and longevity, such as poor housing quality, pollution, and crime (Goldman 2006; Link and Phelan 1995). Individuals' experience of and exposure to multiple psychosocial, environmental, and biomedical risk factors are shaped by SES and race/ethnicity (House 2002). In other words, SES structures social life in ways that impact health and health behavior (Pampel et al. 2010).

Social disadvantage represents the accumulation of experiences and exposures in the material world which have direct implications for health and help to explain the size and persistence of racial/ethnic and socioeconomic health disparities (House 2002; Lynch et al. 2000). For example, individuals of low SES may not only have inadequate resources to purchase health care or eat nutritiously, but they may also live in high crime neighborhoods with poor housing that can have a negative impact on their health and health behaviors. In this way, social disadvantage can be conceptualized as a fundamental cause of poor health outcomes because it involves access to resources and experiences that can either aid or prevent individuals from avoiding diseases/poor health behaviors and their negative consequences through multiple pathways (Link and Phelan 1995).

It is also important to understand that disadvantage occurs at multiple levels of social context. The social-ecological perspective (Bronfenbrenner 1979, 2005; Dahlgren and Whitehead 1991; Glass and McAtee 2006) highlights the fact that individuals operate within multiple and overlapping social contexts that influence multiple individual outcomes, including health, behavior, and development. These social contexts include, but are not limited to, the immediate environment such as the family and larger levels of social context, such as the peer group, workplace, school, and neighborhood. Individuals interact in these multiple and overlapping environments and these environments are strongly linked to one another. In addition, individuals both influence and are influenced by these contexts (Bronfenbrenner 1979, 2005). For these reasons, it is important to examine multiple levels of social context when attempting to understand the relationship between social disadvantage and health. It is also important to note that different social contexts exert stronger or weaker influences on individual level development and 
behavior as individuals move through the life course (Elder 1997, 1998). For example, the school and family environment are very important influences in childhood, and the peer environment and romantic relationships become important social contexts in adolescence (Brown 1990; Giordano 2003). In this article, I will focus on how the social conditions of disadvantage at the individual-, family-, school-, and neighborhoodlevel influence obesity.

\section{Inequality and obesity}

As discussed earlier, among the US population there is considerable socioeconomic inequality in obesity. This means that the populations with the least education and highest poverty rates, and racial/ethnic minorities, also have the highest rates of obesity (Drewnowski and Specter 2004). Many researchers argue multiple aspects of disadvantage at the individual-, household-, school- and neighborhood-level influence obesity through diet and physical activity (or lack thereof) (Pampel et al. 2010).

Although the causes of obesity are complex, at the most basic level, obesity is the result of an imbalance between food and beverage consumption (energy intake) and physical activity (energy expenditure) (Egger and Swinburn 1997). Weight gain occurs when calories consumed from food and beverages exceed calories expended by physical activity and metabolic functions. Therefore, one way to understand how inequality is related to obesity is to examine how social disadvantage influences energy expenditure and energy intake. Below, I will review how different aspects of inequality, at multiple levels, might influence obesity through energy intake and energy expenditure.

\section{Energy intake}

Social disadvantage influences the ability to regulate energy intake (food consumption) because it influences the capacity with which individuals can make healthy food choices. It affects the resources to purchase healthy foods, awareness of healthy eating, and access to healthful foods (Drewnowski and Darmon 2005; Drewnowski and Specter 2004; Lovasi et al. 2009).

Poverty is associated with low food expenditures, lower quality diets, and low fruit and vegetable consumption (Drewnowski and Specter 2004). Low-income groups consume high-fat and energy-dense diets, such as sugars, cereals, potatoes, and processed meat products, because they are more affordable than diets composed of fresh vegetables and fruits and lean meats and fish (Drewnowski and Darmon 2005; Drewnowski and Specter 2004). For example, a person with a limited income will find it more cost effective to buy five packets of macaroni and cheese for five dollars versus one bag of organic mixed green salad for the same price. The macaroni is cheaper than the salad and a larger quantity can be consumed for the same price as a smaller quantity of salad. It is also non-perishable and will last longer than fresh vegetables. Low education has also been linked to less nutritional knowledge. Therefore, because of the lower cost of energy-dense foods, socially disadvantaged individuals and families end up consuming less nutritious and more energy-dense foods which can promote obesity.

In addition, disadvantaged individuals may also suffer from situations where there are episodic food shortages, due to lack of money that occur regularly (e.g., each month). This might be especially the case for individuals on welfare and/or food assistance programs (Dietz 1995; Townsend et al. 2001). When money runs low individuals may restrict their food consumption and then binge on energy-dense food when money is available. This 
type of eating has been described as stress-induced eating, which occurs after 'restrained' eating (Greeno and Wing 1994). This eating cycle of food restriction and overconsumption may also lead to weight gain over time, increasing the risk of obesity (Dietz 1995; Townsend et al. 2001).

Related to episodic food shortages, poor individuals are also at risk of being food insecure (Burns 2004; Ribar and Hamrick 2003; Rose 1999). Food insecurity is defined as uncertain or limited availability of safe and nutritionally adequate foods or uncertain or limited capacity to obtain adequate foods in ways that are socially acceptable (Andersen 1990). Although food insecurity and poverty are related, they can be conceptualized as two separate measures of economic hardship. Not all poor individuals are food insecure and not all food insecure individuals are poor, but both groups face unique, and sometimes related, hardships (Ribar and Hamrick 2003). Several studies have found associations between food insecurity and obesity, with the strongest relationships found for White women (Adams et al. 2003; Alaimo et al. 2001; Casey et al. 2006; Lyons et al. 2008; Townsend et al. 2001; Wilde and Peterman 2006). However, other studies have not found support for this relationship (Gundersen et al. 2009; Rose and Bodor 2006; Whitaker and Sarin 2007). More research is needed to test the hypotheses that increased body weight might be a result of food insecurity, food choices, or physiological adaptations in response to episodic food shortages (Dietz 1995; Meyers et al. 2006; also see Burns 2004 for a review of the literature linking food insecurity to obesity).

Eating behaviors are also influenced by knowledge about diet quality and healthy behaviors as well as socio-cultural norms (Caprio et al. 2008). Lower-educated and lower-income individuals may not have as much awareness of health issues relevant to nutrition and diet and they may also not engage in healthy life-style behaviors (Winkleby et al. 1992). For example, men and women with a college degree are 2.5 times as likely to report that they pay a lot or a fair amount of attention to nutritional information from scientific experts compared to men and women with less than a high school degree (Bleich et al. 2007). Therefore, disadvantaged individuals may consume more energydense foods and beverages due to lack of knowledge of the nutritional adequacy of these foods and/or its influence on body weight.

There are also numerous ways in which culture can influence racial/ethnic disparities in obesity, including ethnic/cultural and socioeconomic differences in ideal body image, perceptions of risk associated with obesity, reduced health care utilization, child-feeding practices, and food consumption (for a review, see Candib 2007; Caprio et al. 2008; DiGioacchino et al. 2001; Kumanyika et al. 2008). Certain cultures might see heavier bodies as symbolizing protection from hunger and physical robustness rather than being unhealthy (Kumanyika 1993). Indeed, in inner-city African-American communities, thinness may actually be associated with poverty, disease, and drug addiction (Wilkinson and Pickett 2009b). Among African-Americans, larger body sizes are more acceptable and obesity is more likely be seen as attractive and desirable, when compared to Whites (Candib 2007; Caprio et al. 2008; Kumanyika et al. 2008). Therefore, food consumption might reflect these ideals.

One way in which culture and inequality may interact to influence obesity outcomes is through differential exposure of nutrition marketing to racial/ethnic groups. Cultural differences in exposure to nutrition marketing may influence racial/ethnic differences in the desirability and motivation to eat unhealthy foods. This might influence obesity, because racial/ethnic groups may be disproportionately targeted for marketing of foods low in nutrient density and high in calories (Caprio et al. 2008). For example, AfricanAmerican children are exposed to 14 percent higher levels of food-related television 
advertising than White children, with the most frequent category of food-related television advertising being fast food (Powell et al. 2007b).

In addition to individual level SES, neighborhood socioeconomic conditions, and related built environment characteristics, may also influence food consumption, by creating food environments that can facilitate or hinder healthy eating practices (Story et al. 2008). The built environment includes human made or modified physical and social features that make up the structure of a community and might influence obesity, such as food sources, housing, and venues for physical activity (Papas et al. 2007). The food environments that characterize the communities, in which low-income and racial/ethnic minorities live, impede their ability to make healthy food choices because healthy foods are not accessible, available, or affordable (Story et al. 2008). Low-income urban areas, with large minority populations, as well as some rural areas, lack large (and chain) super markets, and other food stores that provide affordable healthful food products. In addition, these areas contain large numbers of fast food restaurants and smaller grocery stores or convenience stores, where healthful food products are less likely to be available and/or are more expensive (Larson et al. 2009; Lovasi et al. 2009; Powell et al. 2007a; Small and McDermott 2006). For example, low-income neighborhoods have only 75 percent as many chain supermarkets as middle-income neighborhoods and AfricanAmericans neighborhoods have only 50 percent as many chain supermarkets as White neighborhoods. In addition, Hispanic neighborhoods have only 32 percent as many chain supermarkets as non-Hispanic neighborhoods (Powell et al. 2007a). Taken together, these neighborhood characteristics increase the likelihood of unhealthy food consumption for socially disadvantaged individuals who live there, increasing the likelihood of obesity.

Schools are also a particularly salient social context for children and adolescents. Children spend a large proportion of time in school and consume and expend a large proportion of energy in schools. Previous research has found school income to be related to BMI, with poorer schools being related to higher BMI among adolescents (Richmond and Subramanian 2008). Low-income schools might be related to obesity because they may provide less nutritious foods and have more access to unhealthy snacks and beverages in vending machines, as well as in establishments close to school (Anderson and Butcher 2006b; Neckerman et al. 2010).

\section{Energy expenditure}

Similar to energy intake, social disadvantage influences the ability to regulate energy expenditure (physical activity) because it influences the capacity with which individuals can make healthy decisions regarding regular exercise, particularly leisure time physical activity (i.e., physical activity outside of work and school, such as jogging, running, swimming, and dancing). It affects the resources and time needed to engage in physical activity, awareness of the health benefits of physical activity and access to safe, affordable, and adequate areas to engage in physical activity (King et al. 1992).

Disadvantaged individuals may not have the resources or time to engage in physical activity. Individuals with limited resources may not have the money to allocate to exercise facilities or for their children to be able to engage in organized activities, such as sports, outside of school (Dearing et al. 2009; Sallis et al. 2000). In addition, disadvantaged individuals will have less time, possibly due to inflexible work schedules, child care needs or lack of transportation, to engage in physical activity (Caprio et al. 2008; Pampel et al. 2010; Seedfeldt et al. 2002). Disadvantaged individuals may also be unaware of the 
health benefits of regular physical activity for reducing the likelihood of weight gain. In addition to lacking health knowledge, low SES individuals are less receptive to new health information, such as recommended physical activity guidelines (Link and Phelan 1995; Williams and Jackson 2005).

In addition to the economic barriers to physical activity that are faced by a large proportion of minority and low-income groups, there are also possible cultural barriers that can serve to prevent engagement in regular physical activity. As discussed in the research on energy consumption, cultural attitudes valuing larger body weight, may influence physical activity behavior (Caprio et al. 2008; Seedfeldt et al. 2002). Certain groups may deem physical activity to be unnecessary or inappropriate, especially if it is seen as a tool for weight loss or weight maintenance. Certain cultures or ethnic groups might see physical activity as less important than other attributes, such as academic skills or social competence (Seedfeldt et al. 2002) and elements of physical appearance other than body weight. For example, in a qualitative study examining socio-cultural perspectives on physical activity among African-American and American Indian women, it was found that African-American women saw their hair to be a barrier to physical activity. Hair was considered to be an important element of physical appearance. These women were concerned that if their hair got wet due to perspiration or from swimming, it would ruin their hair style (Henderson and Ainsworth 2000), which required a significant amount of time and money to maintain (Dietz 2001). For African-American women, their hair may be an important part of their identity and marker of beauty and pride (Rooks 1996) that supersedes the health benefits of physical activity.

Minorities and the poor also tend to live in social and physical environments that are not conducive to physical activity because of lack of access to indoor and outdoor places to exercise or walk (such as gyms, pools, parks, playgrounds, and trails), crime-related safety issues, and aesthetic problems (Lovasi et al. 2009). Low-income and minority neighborhoods, particularly African-American neighborhoods, have less access to public and private recreational facilities for exercise, such as health clubs and pools (Sallis and Glanz 2009). Low-income individuals may live in areas where there are poor sidewalk conditions and poor street lighting that inhibit walking or biking. And even when sidewalks and streetlights are available; concerns for safety inhibit outdoor exercise. In addition, poor neighborhoods have higher levels of physical and social disorder, for example, more traffic, noise, and presence of strangers and higher crime rates that influence individuals' perceptions of being unsafe and keep them from exercising outside (Lovasi et al. 2009; McNeill et al. 2006). Parents from low-income families are especially concerned about their children and their perceived safety due to neighborhood conditions which prohibits children's ability to play outside and increases the likelihood they will engage in sedentary behaviors, such as watching TV and playing video games (Carver et al. 2008; Lovasi et al. 2009). It should be noted that, in addition to engaging in lower levels of physical activity, the poor and racial/ethnic minorities, particularly African-Americans, engage in higher levels of leisure time physical inactivity (such as TV viewing) than more advantaged groups (Clarke et al. 2009b; Hanson and Chen 2007; Harris et al. 2006).

As discussed earlier, schools are a salient social context for children and adolescents. Disadvantaged school environments have been linked to lower levels of physical activity among adolescents (Richmond et al. 2006). This relationship could be due to lack of strong physical education programs and organized sports or lack of appropriate and safe recreational facilities that are conducive to physical activity (Sallis and Glanz 2009). 


\section{Summary of energy intake and expenditure research}

In summary, there are multiple ways that inequality at multiple levels of social context can influence obesity outcomes, through food consumption and physical activity. Disadvantaged individuals not only lack personal resources and knowledge that influence energy intake and expenditure, but they are also more likely to live in under-resourced and unsafe environments that limit the ability to engage in healthy behaviors. Cultural factors complicate these relationships and also independently influence health behaviors related to obesity in important ways.

\section{The role of stress in understanding the inequality and obesity relationship}

Another important pathway linking inequality or disadvantaged social position to obesity is stress (Pearlin 1989). Disadvantaged groups might face higher levels of stress due to both economic and other forms of social adversity, such as discrimination, particularly for African-Americans and other racial/ethnic minority groups (Clark et al. 1999; Williams 1999). Previous research has found that higher levels of (chronic) stress are associated with increases in unhealthy coping behaviors, such as smoking, drinking, and physical inactivity, particularly among socially disadvantaged groups (Lantz et al. 2005). Overeating due to stress is usually characterized by the consumption of unhealthy 'comfort foods', such as pizza, hamburgers, and chocolate (Dallman 2009; Dallman et al. 2003; Laitinen et al. 2002). Moreover, recent research has found that African-American adults, due to their disadvantaged social circumstances and experiences with stress, are more likely to engage in these maladaptive coping strategies as a response to high levels of stress, which have negative implications for the incidence of poor physical health conditions (such as CVD and diabetes), but might also be protective of the incidence and severity of poor mental health conditions, such as depression (Jackson et al. 2010; Mezuk et al. 2010).

Gendered coping responses to stress may also help explain why there are larger socioeconomic disparities in obesity among women than men. Previous research has also found that women, particularly African-American women, tend to use (over)eating as a coping strategy for stress, as well as retreating indoors (resulting in higher levels of inactivity and lower levels of physical activity), particularly in unsafe environments (Jackson and Knight 2006). On the other hand, African-American men are more likely to respond to stress by engaging in higher levels of physical activity and drug abuse (Williams 2003).

Moreover, not only does stress influence energy intake and expenditure, but it can also influence hormonal processes that can affect how fat is stored and accumulates in the body. For example, previous research has found that exposure to chronic stress leads to abdominal or central obesity (Björntorp 2001; Drapeau et al. 2003). Abdominal obesity has been linked to CVD and mortality independent of BMI (Zhang et al. 2008). This is just one example of many possible responses to stress occurring inside the body (i.e., pathogenic responses) that can result in obesity (Holmes et al. 2010).

The complex relationship between stress and health and the pathways linking stress to health highlight the fact that energy intake and expenditure cannot alone explain the higher prevalence of obesity among disadvantaged population groups.

\section{Conclusion}

In this review, I explained possible ways that inequality can be linked to obesity via energy intake and expenditure, using the ecological model to examine disadvantage at 


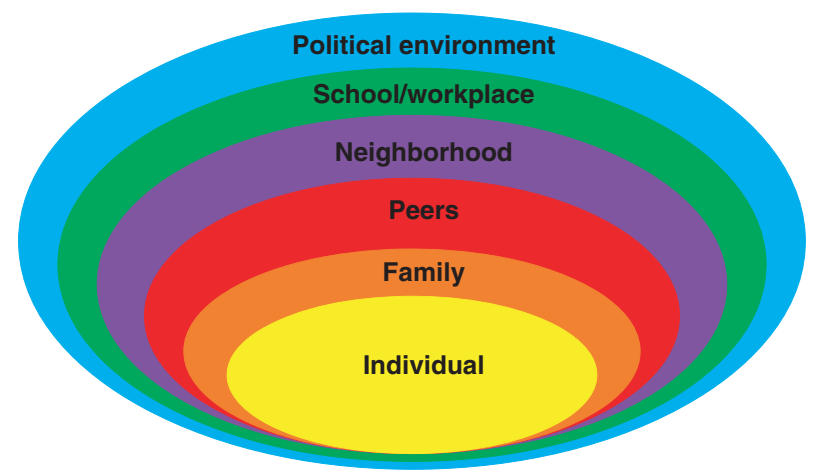

Figure 1. Social-ecological model of multiple levels of contextual disadvantage. Note. Figure adapted from Bronfenbrenner (1979).

the individual-, family-, school-, and neighborhood-level and drawing from theories and evidence from multiple disciplines and perspectives. This review is by no means exhaustive, but does highlight important components of the social and physical environment of disadvantaged individuals that can influence obesity outcomes. (For an important recent review of the link between SES and physical activity, diet and obesity, see Pampel et al. 2010.) It is important to note that other levels of social context also influence health behaviors such as peer groups and other social networks, the workplace and larger institutional and political forces (Fowler and Christakis 2008; McKay et al. 2007). Individuals not only operate within their family, schools, and neighborhoods, but also within these other contexts, which has important implications on obesity outcomes (see Figure 1). Therefore, it is imperative to understand the multiple causes of obesity within a multilevel framework (Huang et al. 2009).

In this review, I have discussed how both SES and race/ethnicity are related to obesity outcomes. It is important to note that race and ethnicity are often confounded with SES. Certain minority groups, such as African-Americans and some Hispanic groups, have higher rates of poverty and live in more disadvantaged areas than Whites (Iceland 2006). In addition to facing higher levels of disadvantage, they also face particular circumstances that increase disadvantage, such as racial segregation and discrimination that can also influence obesity, via influencing the physical and social conditions of neighborhoods or stress (Lovasi et al. 2009; Williams 1992). Some cultural factors that influence obesity outcomes are a result of these forces. For example, in W. E. B. DuBois' (1899) discussion of the conditions of African-Americans in Philadelphia (Pennsylvania) at the turn of the century, he argued that their cultural behaviors, such as their diet and activity, were the result of social and economic forces such as slavery, substandard housing, and under-resourced neighborhoods in racially segregated urban areas where they were forced to reside.

Although only briefly discussed in this review, other research has highlighted biological stress pathways that can lead to increased weight and body fat, disordered eating, and reduced levels of physical activity (Greeno and Wing 1994; Kuo et al. 2008). Socially disadvantaged individuals face multiple stressors that can influence their health, such as unemployment, trouble paying bills, food insecurity, inadequate housing, and neighborhood crime. This is particularly the case for minorities who face additional unique stressors, such as racial discrimination and segregation (Taylor and Repetti 1997). Stress research also highlights the fact that energy consumption and expenditure cannot alone 
explain the relationship between disadvantage and obesity. Understanding these stress pathways is an important avenue for future research. Future research should also continue to investigate gender differences in SES disparities, by determining specific mechanisms that may be driving these differences.

As noted earlier, this review focused on materialist explanations of inequality and its links to obesity, however, psychosocial measures of relative inequality are also an important area of research, particularly in illuminating the role of community levels of trust, social support, and social capital in influencing obesity (Wilkinson and Pickett 2009b). This interesting body of complex and nuanced research which has examined relative measures of inequality as it relates to mortality, mental health, obesity, and other physical health and chronic conditions deserves its own review. For a recent review of this literature as it relates to physical activity, diet, and obesity, see Pampel et al. (2010).

Obesity has increased across all population groups in the United States and is a large public health concern. Factors explaining the rise in obesity and disproportionate prevalence of obesity for poor and minority populations are complex and we don't have all the answers. There is still a lot more that needs to be explained in understanding the mechanisms that link disadvantage to health, and obesity, in particular. Sociologists can add to these efforts by drawing on sociological theories, such as social stratification, status attainment and critical race theory, and using both quantitative and qualitative methods to explain the social causes of obesity. It will also require that sociologists work with scholars across the fields of social sciences, and in public health, medicine, and biology to integrate theories from multiple disciplines, to better understand these complex processes. Effort by all fields is the only way to reduce overall prevalence of obesity, especially among our most disadvantaged populations.

\section{Acknowledgement}

This research was supported by the Robert Wood Johnson Foundation Health \& Society Scholars Program. The author thanks Kathleen Mullan Harris and Eric S. Williams for their helpful feedback. All errors are my own, and the contents of this paper do not necessarily reflect the views of funding agencies.

\section{Short Biography}

Hedwig Lee's research is located at the intersection of sociology, public health, and demography. She has authored and coauthored papers in Population Research and Policy Review, the Journal of Family Issues, the Journal of Negro Education, Sociological Methodology, American Journal of Epidemiology, and Sociological Perspectives. She is broadly interested in inequalities in health and has studied how social contexts of disadvantage in adolescence influence health and other outcomes in adulthood. Her recent work has explored the effects of family poverty on obesity and physical activity in young adulthood, and the influence of early marriage on health outcomes in adulthood. She holds a BS in Policy Analysis from Cornell University and received her $\mathrm{PhD}$ in Sociology from the University of North Carolina at Chapel Hill. She was also awarded an Interdisciplinary Certificate in Health Disparities from the University of North Carolina at Chapel Hill School of Public Health and School of Medicine Program on Ethnicity, Culture and Health Outcomes. She is currently a Robert Wood Johnson Health \& Society Scholar at the University of Michigan and on leave (until Fall 2011) from a position as an Assistant Professor in the Department of Sociology at the University of Washington in Seattle. 


\section{Note}

* Correspondence address: Hedwig Lee, Department of Epidemiology, School of Public Health, University of Michigan, 3644 SPH Tower, 1415 Washington Heights, Ann Arbor, MI 48109-2029, USA. E-mail: hedylee@ umich.edu

\section{References}

Adams, E. J., L. Grummer-Strawn and G. Chavez. 2003. 'Food Insecurity Is Associated With Increased Risk of Obesity in California Women.' Journal of Nutrition 133: 1070-4.

Adler, N. E. and J. M. Ostrove. 1999. 'Socioeconomic Status and Health: What We Know and What We Don't.' Annals of the New York Academy of Sciences 896: 3-15.

Alaimo, K., C. M. Olson and E. A. Frongillo Jr. 2001. 'Low Family Income and Food Insufficiency in Relation to Overweight in Children: Is There a Paradox?' Archives of Pediatric and Adolescent Medicine 155(10): 1161-7.

Andersen, S. A. 1990. 'Core Indicators of Nutritional State for Difficult to Sample Populations.' The Journal of Nutrition 120: 1557S-600S.

Anderson, P. and K. F. Butcher. 2006a. 'Reading, Writing, and Refreshments: Do School Finances Contribute to Childhood Obesity?' Journal of Human Resources 41(3): 467-94.

Anderson, P. M. and K. F. Butcher. 2006b. 'Childhood Obesity: Trends and Potential Causes.' The Future of Children 16(1): 19-45.

Apovian, C. M. 2010 'The Causes, Prevalence, and Treatment of Obesity Revisited in 2009: What Have We Learned So Far?' American Journal of Clinical Nutrition 91: 277S-9S.

Averett, S. and S. Korenman. 1996. 'The Economic Reality of the Beauty Myth.' The Journal of Human Resources 31(2): 304-30.

Ball, K. and D. Crawford. 2005. 'Socioeconomic Status and Weight Change in Adults: A Review.' Social Science E Medicine 60(9): 1987-2010.

Bartley, M. 2004. Health Inequality: An Introduction to Theories, Concepts and Methods. Cambridge, UK: Polity Press.

Beckfield, J. 2004. 'Does Income Inequality Harm Health? New Cross-National Evidence.' Journal of Health and Social Behavior 45: 231-45.

Beckfield, J. and N. Krieger. 2009. 'Epi+Demos+Cracy: Linking Political Systems and Priorities to the Magnitude of Health Inequalities —Evidence, Gaps, and a Research Agenda.' Epidemiologic Reviews 31: 152-77.

Björntorp, P. 2001. 'Do Stress Reactions Cause Abdominal Obesity Comorbidities?' Obesity Reviews 2: 73-86.

Bleich, S., R. Blendon and A. Adams. 2007. 'Trust in Scientific Experts on Obesity: Implications for Awareness and Behavior Change.' Obesity 15(8): 2145-56.

Boardman, J. D., J. M. Saint Onge, R. G. Rogers and J. T. Denney. 2005. 'Race Differentials in Obesity: The Impact of Place.' Journal of Health and Social Behavior 46: 229-43.

Bronfenbrenner, U. 1979. The Ecology of Human Development: Experiments by Nature and Design. Cambridge, MA: Harvard University Press.

Bronfenbrenner, U. 2005. Making Human Beings Human: Bioecological Perspectives on Human Development. Thousand Oaks, CA: Sage Publications.

Brown, B. B. 1990. 'Peer Groups and Peer Cultures.' Pp. 171-96 in At the Threshold: The Developing Adolescent, edited by S. S. Feldman and G. R. Elliott. Cambridge, MA: Harvard University Press.

Burns, C. 2004. A Review of the Literature Describing the Link Between Poverty, Food Insecurity and Obesity With Specific Reference to Australia. VicHealth. http://www.tehotumanawa.org.nz/documents/File/Nutrition\%20General/ A_review_of_the_literature_describing_the_link_between_poverty_food_insecurity_and_obesity_with_specific_ reference_to_Australia.pdf (Last Accessed 18 October 2009).

Candib, L. M. 2007. 'Obesity and Diabetes in Vulnerable Populations: Reflection on Proximal and Distal Causes.' Annals of Family Medicine 5: 547-56.

Caprio, S., S. R. Daniels, A. Drewnowski, F. R. Kaufman, L. A. Palinkas, A. L. Rosenbloom and J. B. Schwimmer. 2008. 'Influence of Race, Ethnicity, and Culture on Childhood Obesity: Implications for Prevention and Treatment: A Consensus Statement of Shaping America's Health and the Obesity Society.' Diabetes Care 31(11): 2211-21.

Carver, A., A. Timperio and D. Crawford. 2008. 'Playing it Safe: The Influence of Neighbourhood Safety on Children's Physical Activity-A Review.' Health and Place 14(20): 217-27.

Casey, P. H., P. M. Simpson, J. M. Gossett, M. L. Bogle, C. M. Champagne, C. Connell, D. Harsha, B. McCabeSellers, J. M. Robbins, J. E. Stuff and J. Weber. 2006. 'The Association of Child and Household Food Insecurity With Childhood Overweight Status.' Pediatrics 118(5): e1406-13.

Cawley, J. 2004. 'The Impact of Obesity on Wages.' The Journal of Human Resources 39(2): 451-74.

Center for Disease Control (CDC). 2008. Prevalence of Overweight, Obesity and Extreme Obesity Among Adults: United States, Trends 1960-62 Through 2005-2006. NCHS Health E-Stat. Hyattsville, MD: US Department of Health 
and Human Services, CDC, National Center for Health Statistics. http://www.cdc.gov/nchs/data/hestat/ overweight/overweight_adult.htm (Last Accessed 18 October 2010).

Center for Disease Control (CDC). 2009. Overweight and Obesity: Health Consequences. Hyattsville, MD: US Department of Health and Human Services, CDC, National Center for Health Statistics. http://www.cdc.gov/obesity/ causes/health.html (Last Accessed 18 October 2010).

Chang, V. W. and N. A. Christakis. 2005. 'Income Inequality and Weight Status in US Metropolitan Areas.' Social Science \& Medicine 61: 83-96.

Clark, R., N. B. Anderson, V. R. Clark and D. R. Williams. 1999. 'Racism as a Stressor for African Americans: A Biopsychosocial Model.' American Psychologist 54: 805-16.

Clarke, P., P. M. O’Malley, L. D. Johnston and J. E. Schulenberg. 2009a. 'Social Disparities in BMI Trajectories Across Adulthood by Gender, Race/Ethnicity and Lifetime Socio-Economic Position: 1986-2004.' International Journal of Epidemiology 38: 499-509.

Clarke, P. J., P. M. O'Malley, L. D. Johnston, J. E. Schulenberg and P. Lantz. 2009b. 'Differential Trends in Weight-Related Health Behaviors Among American Young Adults by Gender, Race/Ethnicity, and Socioeconomic Status: 1984-2006.' American Journal of Public Health 99: 1893-901.

Crosnoe, R. 2007. 'Gender, Obesity, and Education.' Sociology of Education 80: 241-60.

Cummins, S. and S. McIntyre. 2006. 'Food Environments and Obesity-Neighborhood or Nation?' International Journal of Epidemiology 35: 100-4.

Dahlgren, G. and M. Whitehead. 1991. Policies and Strategies to Promote Social Equity in Health. Stockholm: Institute for Futures Studies.

Dallman, M. F. 2009. 'Stress-Induced Obesity and the Emotional Nervous System.' Trends in Endocrinology and Metabolism 21(3): 159-65.

Dallman, M. F., N. Pecoraro, S. F. Akana, S. E. la Fleur, F. Gomez, H. Houshyar, M. E. Bell, S. Bhatnagar, K. D. Laugero and S. Manalo. 2003. 'Chronic Stress and Obesity: A New View of "Comfort Food.'.' Proceedings of the National Academy of Sciences 100(20): 11696-701.

Daniels, S. R. 2006. 'The Consequences of Childhood Overweight and Obesity.' Future of Children 16(1): 47-67.

Dearing, E., C. Wimer, S. D. Simpkins, T. Lund, S. M. Bouffard, P. Caronongan, H. Kreider and H. Weiss. 2009. 'Do Neighborhood and Home Contexts Help Explain Why Low-Income Children Miss Opportunities to Participate in Activities Outside of School?' Developmental Psychology 45(6): 1545-62.

Dietz, W. H. 1995. 'Does Hunger Cause Obesity?' Pediatrics 95(5): 766-7.

Dietz, W. H. 2001. 'Focus Group Data Pertinent to the Prevention of Obesity in African Americans.' American Journal of the Medical Sciences 322(5): 275-8.

DiGioacchino, R. F., R. G. Sargent and M. Topping. 2001. 'Body Dissatisfaction Among White and African American Male and Female College Students.' Eating Behaviors 2: 39-50.

Dor, A., C. Ferguson, E. Tan and C. Langwith. 2010. A Heavy Burden: The Individual Costs of Being Overweight and Obese in the United States. George Washington University School of Public Health and Health Service. http:// www.gwumc.gwu.edu/sphhs/departments/healthpolicy/dhp_publications/pub_uploads/dhpPublication_35308C475056-9D20-3DB157B39AC53093.pdf (Last Accessed 21 September 2010)

Drapeau, V., F. Therrien, D. Richard and A. Tremblay. 2003. 'Is Visceral Obesity and Physiological Adaptation to Stress?' Panminerva Medica 45(3): 189-95.

Drewnowski, A. and N. Darmon. 2005. 'The Economics of Obesity: Dietary Energy Density and Energy Cost.' American Journal of Clinical Nutrition 82(1): 265S-73S.

Drewnowski, A. and S. E. Specter. 2004. 'Poverty and Obesity: The Role of Energy Density and Energy Costs.' American Journal of Clinical Nutrition 79: 6-16.

DuBois, W. E. B. 1899. The Philadelphia Negro: A Social Study. Philadelphia, PA: University of Pennsylvania Press.

Egger, G. and B. Swinburn. 1997. 'An “Ecological”' Approach to the Obesity Pandemic.' British Medical Journal 315: 477-80.

Elder, G. H., Jr. 1997. 'The Life Course and Human Development.' Pp. 939-91 in Handbook of Child Psychology, Volume 1: Theoretical Models of Human Development, edited by R. M. Lerner and W. Damon. New York, NY: Wiley.

Elder, G. H., Jr. 1998. 'The Life Course as Developmental Theory.' Child Development 69: 1-12.

Elo, I. T. 2009. 'Social Class Differentials in Health and Mortality: Patterns and Explanations in Comparative Perspective.' Annual Review of Sociology 35: 553-72.

Feinstein, J. S. 1993. 'The Relationship Between Socioeconomic Status and Health: A Review of the Literature.' The Milbank Quarterly 71: 279-322.

Finkelstein, E. A., C. J. Ruhm and K. M. Kosa. 2005. 'Economic Causes and Consequences of Obesity.' Annual Review of Public Health 26: 239-57.

Flegal, K. M., M. D. Carroll, C. L. Ogden and L. R. Curtin. 2010. 'Prevalence and Trends in Obesity Among US Adults, 1999-2008.' Journal of American Medical Association 303(3): 235-41.

Fowler, J. H. and N. A. Christakis. 2008. 'Estimating Peer Effects on Health in Social Networks: A Response to Cohen-Cole and Fletcher; and Trogdon, Nonnemaker, and Pais.' Journal of Health Economics 27: 1400-5. 
Giordano, P. C. 2003. 'Relationships in Adolescence.' Annual Review of Sociology I: 257-81.

Glass, T. A. and M. J. McAtee. 2006. 'Behavioral Science at the Crossroads in Public Health: Extending Horizons, Envisioning the Future.' Social Science and Medicine 62(7): 1650-71.

Goldman, N. 2006. 'Social Inequalities in Health: Disentangling the Underlying Mechanisms.' Annals of the New York Academy of Sciences 954: 118-39.

Goodman, E. 2003. 'Letting the "Gini”' Out of the Bottle: Social Causation and the Obesity Epidemic.' The Journal of Pediatrics 142(3): 228-30.

Gortmaker, S. L., A. Must, J. M. Perrin, A. M. Sobol and W. H. Dietz. 1993. 'Social and Economic Consequences of Overweight in Adolescence and Young Adulthood.' The New England Journal of Medicine 329(14): 1008-12.

Greeno, C. G. and R. R. Wing. 1994. 'Stress-Induced Eating.' Psychological Bulletin 115(3): 444-64.

Gundersen, C., S. Garasky and B. J. Lohman. 2009. 'Food Insecurity Is Not Associated With Childhood Obesity as Assessed Using Multiple Measures of Obesity1-3.' The Journal of Nutrition 139(6): 1173-8.

Hanson, M. D. and E. Chen. 2007. 'Socioeconomic Status, Race, and Body Mass Index: The Mediating Role of Physical Activity and Sedentary Behaviors During Adolescence.' Journal of Pediatric Psychology 32(3): 250-9.

Harper, S. and J. Lynch. 2007. 'Trends in Socioeconomic Inequalities in Adult Health Behaviors Among U.S. States, 1990-2004.' Public Health Reports 122: 177-89.

Harris, K. M., P. Gordon-Larsen, K. Chantala and J. R. Udry. 2006. 'Longitudinal Trends in Race/Ethnic Disparities in Leading Health Indicators From Adolescence to Young Adulthood.' Archives of Pediatrics and Adolescent Medicine 160: 74-81.

Henderson, K. A. and B. E. Ainsworth. 2000. 'Sociocultural Perspectives on Physical Activity in the Lives of Older African American and American Indian Women: A Cross Cultural Activity Participation Study.' Women and Health 31(1): 1-20.

Holmes, M. E., P. Ekkekakis and J. C. Eisenmann. 2010. 'The Physical Activity, Stress, and Metabolic Syndrome Triangle: A Guide to Unfamiliar Territory for the Obesity Researcher.' Obesity Reviews 11(7): 492-507.

House, J. S. 2002. 'Understanding Social Factors and Inequalities in Health: $20^{\text {th }}$ Century Progress and $21^{\text {st }}$ Century Prospects.' Journal of Health and Social Behavior 43(2): 125-42.

Huang, T. T., A. Drewnowski, S. K. Kumanyika and T. A. Glass. 2009. 'A Systems-Oriented Multi-Level Framework for Addressing Obesity in the 21st Century.' Preventing Chronic Disease 6(3): A82.

Iceland, J. 2006. Poverty in America (2nd Edition). Berkeley, CA: University of California Press.

Jackson, J. S. and K. M. Knight. 2006. 'Race and Self-Regulatory Health Behaviors: The Role of the Stress Response and the HPA Axis in Physical and Mental Health Disparities.' Pp. 189-208 in Social Structure Aging and Self-Regulation in the Elderly, edited by K. W. Schaie and L. S. Carstensen. New York, NY: Springer Publishing Company.

Jackson, J. S., K. M. Knight and J. A. Rafferty. 2010. 'Race and Unhealthy Behaviors: Chronic Stress, the HPA Axis, and Physical and Mental Health Disparities Over the Life Course.' American Journal of Public Health 100: 933-9.

Janssen, I., W. F. Boyce, K. Simpson and W. Pickett. 2006. 'Influence of Individual- and Area-Level Measures of Socioeconomic Status and Obesity, Unhealthy Eating, and Physical Inactivity in Canadian Adolescents.' American Journal of Clinical Nutrition 83: 139-45.

Kawachi, I. and B. P. Kennedy. 1997a. 'Health and Social Cohesion: Why Care About Income Inequality?' British Medical Journal 314(7086): 1037-40.

Kawachi, I. and B. P. Kennedy. 1997b. 'The Relationship of Income Inequality to Mortality: Does the Choice of Indicator Matter?' Social Science and Medicine 45(7): 1121-7.

Kawachi, I. and B. P. Kennedy. 1999. 'Income Inequality and Health: Pathways and Mechanisms.' Health Services Research 34(1): 215-27.

Kima, D., I. Kawachi, S. Vander Hoornb and M. Ezzatic. 2008. 'Is Inequality at the Heart of It? Cross-Country Associations of Income Inequality With Cardiovascular Diseases and Risk Factors.' Social Science and Medicine 66(8): 1719-32.

King, A. C., S. N. Blair, D. E. Bild, R. K. Dishman, P. M. Dubbert, B. H. Marcus, N. B. Oldridge, R. S. Pafffenbarger Jr., K. E. Powell and K. K. Yeager. 1992. 'Determinants of Physical Activity and Interventions in Adults.' Medicine and Science in Sports and Exercise 24(6): 221-36.

Kondo, N., G. Sembajwe, I. Kawachi, R. M. Van Dam, S. V. Subramanian and Z. Yamagata. 2009. 'Income Inequality, Mortality, and Self Rated Health: Meta-Analysis of Multilevel Studies.' British Medical Journal 339: b4471.

Kumanyika, S. and S. Grier. 2006. 'Targeting Interventions for Ethnic Minority and Low-Income Populations.' The Future of Children 16(1): 187-207.

Kumanyika, S. K. 1993. 'Special Issues Regarding Obesity in Minority Populations.' Annals of Internal Medicine 119(7): 650-4.

Kumanyika, S. K., E. Obarzanek, N. Stettler, R. Bell, A. E. Field, S. P. Fortmann, B. A. Franklin, M. W. Gillman, C. E. Lewis, W. C. Poston II, J. Stevens and Y. Hong. 2008. 'Population-Based Prevention of Obesity. The 
Need for Comprehensive Promotion of Healthful Eating, Physical Activity, and Energy Balance.' Circulation 118: 428-64.

Kuo, L. E., M. Czarnecka, J. B. Kitlinska, J. U. Tilan, R. Kvetňanský and Z. Zukowska. 2008. 'Chronic Stress, Combined With a High-Fat/High-Sugar Diet, Shifts Sympathetic Signaling Toward Neuropeptide Y and Leads to Obesity and the Metabolic Syndrome.' Annals of the New York Academy of Sciences 1148: 232-7.

Laitinen, J., E. Ek and U. Sovio. 2002. 'Stress-Related Eating and Drinking Behavior and Body Mass Index and Predictors of This Behavior.' Preventive Medicine 34(1): 29-39.

Lantz, P. M., J. S. House, R. P. Mero and D. R. Williams. 2005. 'Stress, Life Events and Socioeconomic Disparities in Health: Results From the Americans' Changing Lives Study.' Journal of Health and Social Behavior 46(3): $274-88$.

Larson, N. I., M. T. Story and M. C. Nelson. 2009. 'Neighborhood Environments: Disparities in Access to Healthy Foods in the U.S.' American Journal of Preventive Medicine 36(1): 74-81.

Lee, H., K. M. Harris and P. Gordon-Larsen. 2009. 'Life Course Perspectives on the Links Between Poverty and Obesity During the Transition to Young Adulthood.' Population Research and Policy Review 28: 505-32.

Lemeshow, A. R., L. Fisher, E. Goodman, I. Kawachi, C. S. Berkey and G. A. Colditz. 2008. 'Subjective Social Status in the School and Change in Adiposity in Female Adolescents: Findings From a Prospective Cohort Study.' Archives of Pediatrics and Adolescent Medicine 162(1): 23-8.

Link, B. G. and J. Phelan. 1995. 'Social Conditions AS Fundamental Causes of Disease.' Journal of Health and Social Behavior 35: 80-94.

Lovasi, G. S., M. A. Hutson, M. Guerra and K. M. Neckerman. 2009. 'Built Environments and Obesity in Disadvantaged Populations.' Epidemiologic Reviews 31: 7-20.

Lutfey, K. and J. Freese. 2005. 'Toward Some Fundamentals of Fundamental Causality: Socioeconomic Status and Health in the Routine Clinic Visit for Diabetes.' American Journal of Sociology 110(5): 1326-72.

Lynch, J. 2000. 'Income Inequality and Health: Expanding the Debate.' Social Science and Medicine 51: 1001-5.

Lynch, J. W. and G. A. Kaplan. 1997. 'Understanding How Inequality in the Distribution of Income Affects Health.' Journal of Health Psychology 2: 297-314.

Lynch, J. W., G. D. Smith, G. A. Kaplan and J. S. House. 2000. 'Income Inequality and Mortality: Importance to Health of Individual Income, Psychosocial Environment, or Material Conditions.' British Medical Journal 320: 1200-4.

Lyons, A., J. Park and C. H. Nelson. 2008. 'Food Insecurity and Obesity: A Comparison of Self-Reported and Measured Height and Weight.' American Journal of Public Health 98(4): 751-7.

Marmot, M. 2004. The Status Syndrome: How Social Standing Affects Our Health and Longevity. New York, NY: Henry Holt and Company.

Massey, D and N. Denton. 1993. American Apartheid. Cambridge, MA: Harvard University Press.

McKay, C. M., B. A. Bell-Ellison, K. Wallace and J. M. Ferron. 2007. 'A Multilevel Study of the Associations Between Economic and Social Context, Stage of Adolescence, and Physical Activity and Body Mass Index.' Pediatrics 119: S84-91.

McLaren, L. 2007. 'Socioeconomic Status and Obesity.' Epidemiologic Reviews 29(1): 29-48.

McNeill, L. H., M. W. Kreuter and S. V. Subramanian. 2006. 'Social Environment and Physical Activity: A Review of Concepts and Evidence.' Social Science and Medicine 63: 1011-22.

Meyers, A. F., R. J. Karp and J. G. Kral. 2006. 'Poverty, Food Insecurity, and Obesity in Children.' Pediatrics 118(5): 2265a-6a.

Mezuk, B., J. A. Rafferty, K. N. Kershaw, D. Hudson, C. M. Abdou, H. Lee, W. E. Eaton and J. S. Jackson. 2010. 'Reconsidering the Role of Social Disadvantage in Physical and Mental Health: Stressful Life Events, Health Behaviors, Race and Depression.' American Journal of Epidemiology 172: 1238-49.

Miech, R. A., S. K. Kumanyika, N. Stettler, B. G. Link, J. C. Phelan and V. W. Chang. 2006. 'Trends in the Association of Poverty With Overweight Among US Adolescents, 1971-2004.' Journal of the American Medical Association 295: 2385-93.

Mokdad, A. H., J. S. Marks, D. F. Stroup and J. L. Gerberding. 2004. 'Actual Causes of Death in the United States, 2000.' The Journal of the American Medical Association 291: 1238-45.

Molarius, A., J. C. Seidell, S. Sans, J. Tuomilehto and K. Kuulasmaa. 2000. 'Educational Level, Relative Body Weight, and Changes in Their Association Over 10 Years: An International Perspective From the WHO MONICA Project.' American Journal of Public Health 90(8): 1260-8.

Morland, K., S. Wing and A. Diez Roux. 2002. 'The Contextual Effect of the Local Food Environment on Residents' Diets: The Atherosclerosis Risk in Communities Study.' American Journal of Public Health 92: 1761-7.

Mujahid, M. S., A. V. Diez Roux, L. N. Borrell and F. J. Nieto. 2005. 'Cross-Sectional and Longitudinal Associations of BMI With Socioeconomic Characteristics.' Obesity Research 13(8): 1412-21.

Neckerman, K. M., M. D. M. Bader, C. A. Richards, M. Purciel, J. W. Quinn, J. S. Thomas, W. Caitlin, C. C. Weiss, G. S. Lovasi and A. Rundle. 2010. 'Disparities in the Food Environments of New York City Public Schools.' American Journal of Preventative Medicine 39(3): 195-202.

Ogden, C. L. and M. D. Carroll. 2010. Prevalence of Obesity Among Children and Adolescents: United States, Trends 1963-1965 Through 2007-2008. NCHS Health E-Stat. Hyattsville, MD: US Department of Health and Human 
Services, CDC, National Center for Health Statistics. http://www.cdc.gov/nchs/data/hestat/obesity_child_ 07_08/obesity_child_07_08.htm (Last Accessed 18 October 2010).

Ogden, C. L., M. D. Carroll, L. R. Curtin, M. M. Lamb and K. M. Flegal. 2010a. 'Prevalence of High Body Mass Index in US Children and Adolescents, 2007-2008.' Journal of the American Medical Association 303(3): 242-9.

Ogden, C. L., M. M. Lamb, M. D. Carroll and K. M. Flegal. 2010b. Obesity and Socioeconomic Status in Children: United States, 2005-2008. NCHS Data Brief No. 15. Hyattsville, MD: US Department of Health and Human Services, CDC, National Center for Health Statistics. http://www.cdc.gov/nchs/data/databriefs/db51.pdf (Last Accessed 6 January 2011).

Organisation for Economic Co-Operation and Development (OECD). 2010. Obesity and the Economics of Prevention: Fit not Fat. OECD Publishing. http://www.oecd-ilibrary.org/social-issues-migration-health/obesity-and-theeconomics-of-prevention_9789264084865-en (Last Accessed 18 October 2010).

Pampel, F. C., P. M. Krueger and J. T. Denney. 2010. 'Socioeconomic Disparities in Health Behaviors.' Annual Review of Sociology 36: 349-70.

Papas, M. A., A. J. Alberg, R. Ewing, K. J. Helzlsouer, T. L. Gary and A. C. Klassen. 2007. 'The Built Environment and Obesity.' Epidemiologic Reviews 29(1): 129-43.

Pearlin, L. I. 1989. 'The Sociological Study of Stress.' Journal of Health and Social Behavior 30: 241-56.

Philipson, T. and R. Posner. 2008. 'Is the Obesity Epidemic a Public Health Problem? A Decade of Research on the Economics of Obesity.' NBER Working Paper No. 14010, Cambridge, MA.

Pickett, K. E., S. Kelly, E. Brunner, T. Lobstein and R. G. Wilkinson. 2005. 'Wider Income Gaps, Wider Waistbands? An Ecological Study of Obesity and Income Inequality.' Journal of Epidemiology and Community Health 59: 670-4.

Powell, L. M., S. Slaterb, D. Mirtchevaa, Y. Baoa and F. J. Chaloupkaa. 2007a. 'Food Store Availability and Neighborhood Characteristics in the United States.' Preventive Medicine 44(3): 189-95.

Powell, L. M., G. Szczypka and F. J. Chaloupka. 2007b. 'Adolescent Exposure to Food Advertising on Television.' American Journal of Preventive Medicine 33(4): S251-6.

Puhl, R. M. and C. A. Heuer. 2009. 'The Stigma of Obesity: A Review and Update.' Obesity 17(5): 941964.

Ribar, D. C. and K. S. Hamrick. 2003. Dynamics of Poverty and Food Sufficiency. Food Assistance and Nutrition Research Report No. (FANRR36). http://www.ers.usda.gov/Publications/FANRR36/ (Last Accessed 18 October 2009).

Richmond, T. K., R. A. Hayward, S. Gahagan, A. E. Field and M. Heisler. 2006. 'Can School Income and Racial/Ethnic Composition Explain the Racial/Ethnic Disparity in Physical Activity Participation?’ Pediatrics 17: 2158-66.

Richmond, T. K. and S. V. Subramanian. 2008. 'School Level Contextual Factors are Associated With the Weight Status of Adolescent Males and Females.' Obesity 16: 1324-30.

Robert, S. A. 1999. 'Socioeconomic Position and Health: The Independent Contribution of Community Socioeconomic Context.' Annual Review of Sociology 5: 489-516.

Robert, S. A. and J. S. House. 1994. 'Socioeconomic Status and Health Across the Life Course.' Pp. 253-74 in Aging and Quality of Life, edited by R. P. Abeles, H. C. Gift and M. G. Ory. New York, NY: Springer.

Robinson, W. R., P. Gordon-Larsen, J. S. Kaufman, C. M. Suchindran and J. Stevens. 2009. 'The Female-Male Disparity in Obesity Prevalence Among Black American Young Adults: Contributions of Sociodemographic Characteristics of the Childhood Family.' American Journal of Clinical Nutrition 89: 1204-12.

Rooks, N. M. 1996. Hair Raising: Beauty, Culture, and African American Women. New Brunswick, NJ: Rutgers University Press.

Rose, D. 1999. 'Economic Determinants and Dietary Consequences of Food Insecurity in the United States.' Journal of Nutrition 129: 517-20.

Rose, D. and J. N. Bodor. 2006. 'Household Food Insecurity and Overweight Status in Young School Children: Results From the Early Childhood Longitudinal Study.' Pediatrics 117(2): 464-73.

Sallis, J. F. and K. Glanz. 2009. 'Physical Activity and Food Environments: Solutions to the Obesity Epidemic.' The Milbank Quarterly 87(1): 123-54.

Sallis, J. F., J. J. Prochaska and W. C. Taylor. 2000. 'A Review of the Correlates of Physical Activity of Children and Adolescents.' Medicine and Science in Sports and Exercise 32(5): 963-75.

Sánchez-Vaznaugh, E. V., I. Kawachi, S. V. Subramanian, B. N. Sánchez and D. Acevedo-Garcia. 2009. 'Do Socioeconomic Gradients in Body Mass Index Vary by Race/Ethnicity, Gender, and Birthplace?' American Journal of Epidemiology 169(9): 1102-12.

Scharoun-Lee, M., J. S. Kaufman, B. M. Popkin and P. Gordon-Larsen. 2009. 'Obesity, Race/Ethnicity and Life Course Socioeconomic Status Across the Transition From Adolescence to Adulthood.' Journal of Epidemiology and Community Health 63: 133-9.

Seedfeldt, V., R. M. Malina and M. A. Clark. 2002. 'Factors Affecting Levels of Physical Activity in Adults.' Sports Medicine 32(3): 143-68.

Senese, L. C., N. D. Almeida, A. K. Fath, B. T. Smith and E. B. Loucks. 2009. 'Associations Between Childhood Socioeconomic Position and Adulthood Obesity.' Epidemiologic Reviews 31: 21-51. 
Small, M. L. and M. McDermott. 2006. 'The Presence of Organizational Resources in Poor Urban Neighborhoods: An Analysis of Average and Contextual Effects.' Social Forces 84(3): 1697-724.

Sobal, J. and A. J. Stunkard. 1989. 'Socioeconomic Status and Obesity: A Review of the Literature.'Psychological Bulletin 105: 260-75.

Shrewsbury, V. and J. Wardle. 2008. 'Socioeconomic Status and Adiposity in Childhood: A Systematic Review of Cross-sectional Studies 1990-2005.' Obesity 16: 275-84.

Story, M., K. M. Kaphingst, R. Robinson-O'Brien and K. Glanz. 2008. 'Creating Healthy Food and Eating Environments: Policy and Environmental Approaches.' Annual Review of Public Health 29: 253-72.

Swallen, K. C., E. N. Reither, S. A. Haas and A. M. Meier. 2005. 'Overweight, Obesity, and Health-Related Quality of Life Among Adolescents: The National Longitudinal Study of Adolescent Health.' Pediatrics 115(2): 340-7.

Taylor, S. E. and R. L. Repetti. 1997. 'Health Psychology: What is an Unhealthy Environment and How Does It Get Under the Skin?' Annual Review of Psychology 48: 411-47.

Townsend, M. S., J. Peerson, B. Love, C. Achterberg and S. P. Murphy. 2001. 'Food Insecurity is Positively Related to Overweight in Women.' Journal of Nutrition 131: 1738-45.

Townsend, P., N. Davidson and M. Whitehead (eds) 1992. Inequalities in Health: The Black Report and the Health Divide. New York: Penguin.

U.S. Department of Health and Human Services (USHHS). 2001. The Surgeon General's Call to Action to Prevent and Decrease Obesity. Rockville, MD: US Department of Health and Human Services, US Public Health Service, Office of the Surgeon General. http://www.surgeongeneral.gov/topics/obesity/calltoaction/CalltoAction.pdf (Last Accessed 6 January 2009).

Viner, R. M. and T. J. Cole. 2005. 'Adult Socioeconomic, Educational, Social, and Psychological Outcomes of Childhood Obesity: A National Birth Cohort Study.' British Journal of Medicine 330(7504): 1354.

Wang, Y. and M. A. Beydoun. 2007. 'The Obesity Epidemic in the United States-Gender, Age, Socioeconomic, Racial/Ethnic, and Geographic Characteristics: A Systematic Review and Meta-Regression Analysis.' Epidemiologic Reviews 29(1): 6-28.

Whitaker, R. C. and A. Sarin. 2007. 'Change in Food Security Status and Change in Weight Are Not Associated in Urban Women With Preschool Children.' Journal of Nutrition 137: 2134-9.

Wickrama, T. K. A., K. A. S. Wickrama and C. M. Bryant. 2006. 'Community Influence on Adolescent Obesity: Race/Ethnic Differences.' Journal of Youth and Adolescence 35(4): 641-51.

Wilde, P. E. and J. N. Peterman. 2006. 'Individual Weight Change Is Associated With Household Food Security Status.' Journal of Nutrition 136: 1395-400.

Wilkinson, R. G. 1992. 'Income Distribution and Life Expectancy.' British Medical Journal 304: 165-8.

Wilkinson, R. G. 1996. Unhealthy Societies: The Afflictions of Inequality. London, UK: Routledge.

Wilkinson, R. G. and K. E. Pickett. 2006. 'Income Inequality and Population Health: A Review and Explanation of the Evidence.' Social Science and Medicine 62(7): 1768-84.

Wilkinson, R. G. and K. E. Pickett. 2007. 'The Problems of Relative Deprivation: Why Some Societies Do Better Than Others.' Social Science and Medicine 65(9): 1965-78.

Wilkinson, R. G. and K. E. Pickett. 2009a. 'Income Inequality and Social Dysfunction.' Annual Review of Sociology 35: 493-511.

Wilkinson, R. G. and K. E. Pickett. 2009b. The Spirit Level: Why Greater Equality Makes Societies Stronger. New York, NY: Bloomsbury Press.

Williams, D. R. 1992. 'Black-White Differences in Blood Pressure: The Role of Social Factors.' Ethnicity and Disease 2: $126-41$.

Williams, D. R. 1999. 'Race, Socioeconomic Status, and Health: The Added Effects of Racism and Discrimination.' Annals of the New York Academy of Sciences 896: 173-88.

Williams, D. R. 2003. 'The Health of Men: Structured Inequalities and Opportunities.' American Journal of Public Health 9: 724-31.

Williams, D. R. and C. Collins. 2001. 'Racial Residential Segregation: A Fundamental Cause of Racial Disparities in Health.' Public Health Reports 116(5): 404-16.

Williams, D. R. and P. B. Jackson. 2005. 'Social Sources of Racial Disparities In Health.' Health Affairs 24(2): 325-34.

Winkleby, M. A., D. E. Jatulis, E. Frank and S. E. Fortmann. 1992. 'Socioeconomic Status and Health: How Education, Income, and Occupation Contribute to Risk Factors for Cardiovascular Disease.' American Journal of Public Health 82: 816-20.

Zhang, C., K. M. Rexrode, R. M. van Dam, T. Y. Li and F. B. Hu. 2008. 'Abdominal Obesity and the Risk of All-Cause, Cardiovascular, and Cancer Mortality Sixteen Years of Follow-Up in US Women.' Circulation 117: 1658-67.

Zhang, Q. and Y. Wang. 2004. 'Socioeconomic Inequality of Obesity in the United States: Do Gender, Age, and Ethnicity Matter?’ Social Science and Medicine 58(6): 1171-80.

Ziol-Guest, K. M., G. J. Duncan and A. Kalil. 2009. 'Early Childhood Poverty and Body Mass Index.' American Journal of Public Health 99(3): 527-32. 